\title{
Estudio de los estilos de enseñanza-aprendizaje desde la perspectiva de los tres agentes educativos: alumnos, docentes y familiares Study of teaching-learning styles from the perspective of the three educational agents: students, teachers and families \\ *Carlos Bou-Sospedra, ${ }^{* *}$ María Huertas González-Serrano, **Mario Alguacil Jiménez \\ *Universitat Jaume I (España), **Universidad Católica de Valencia San Vicente Mártir (España)
}

Resumen. Los estilos de enseñanza-aprendizaje son un aspecto fundamental para garantizar el correcto aprendizaje de los alumnos. Es por ello, que los objetivos de este estudio son conocer los estilos de enseñanza-aprendizaje de los tres agentes educativos (alumnos, profesores y familiares), y analizar qué factores influyen en las puntuaciones de los distintos estilos de enseñanza-aprendizaje de estos tres agentes educativos. Para ello, la muestra está compuesta por un total de 198 estudiantes de secundaria de un centro educativo de Castellón, 23 docentes que impartían clase a estos alumnos, y 104 padres y madres de estos alumnos. Los datos fueron recopilados a través de cuestionarios. Los resultados muestran que alumnos prefieren un estilo teórico, existiendo diferencias significativas entre la preferencia de los estilos según género, gusto por la Educación Física, y notas en esta materia $(p<.05)$. Los profesores más jóvenes se decantan por un estilo de enseñanza activo, mientras que los más mayores suelen utilizar un estilo más estructurado, no influyendo su experiencia laboral ( $p>.05)$. El $49 \%$ de familiares prefieren un estilo más reflexivo en los centros, encontrándose relación entre la tendencia hacia la innovación y qué consideran importante en el aula, con los diferentes estilos $(p<.05)$. Por tanto, los resultados señalan la discordancia en el proceso de enseñanza-aprendizaje entre los diferentes agentes educativos. Es por ello, que los centros educativos deberían conocer su propia la realidad con el fin de mejorar el proceso la adquisición de conocimientos y, en consecuencia, el rendimiento académico de los alumnos/as.

Palabras clave: enseñanza, aprendizaje, educación secundaria, estudiantes, docentes, familias, educación física.

\begin{abstract}
The teaching and learning styles are a fundamental aspect to guarantee the correct learning of the students. Therefore, the objectives of this study are to understand the teaching-learning styles of the three educational agents (students, teachers and family members), and to analyse which factors influence the scores of the different teaching-learning styles of these three educational agents. To this end, the sample is composed of a total of 198 secondary school students from a high school in Castellon, 23 teachers who taught these students, and 104 parents of these students. The data were collected in all cases through questionnaires. The students preferred a theoretical style, and there were significant differences between the preference of styles according to gender, taste for Physical Education, and grades in this subject $(\mathrm{p}<.05)$. Younger teachers opt for an active teaching style, while older ones tend to use a more structured style, not influencing their work experience ( $p>05) .49 \%$ of family members prefer a more reflexive style in the educational centers, finding a relationship between the tendency towards innovation and what they consider important in the classroom, with the different styles $(\mathrm{p}<.05)$. Therefore, the results point out the discordance in the teaching-learning process between the different educational agents. Therefore, the educational centers should know their own reality. All this, with the aim of improving the process of knowledge acquisition and, consequently, the academic performance of the students.
\end{abstract}

Keywords: learning style, teaching style, secondary school, students, teachers, families, physical education.

\section{Introducción}

El rendimiento académico de los alumnos españoles muestra valores inferiores a la media OECD, reflejada en el informe PISA, desde el año 2000 (OECD, 2017). En consecuencia, el estado español ha realizado constantes reformas en la ley educativa con el objetivo de desarrollar un nivel académico alto. Sin embargo, frecuentemente se centran en los contenidos y se olvida la interrelación didáctica, sin tener en cuenta a los agentes educativos y, en consecuencia, los estilos de enseñanza de los docentes o los estilos de aprendizaje de cada alumno (Gil et al., 2007). El aprendizaje se fundamenta en procesos biológicos y psicológicos llevados a cabo en la corteza cerebral y, a través del pensamiento, provocan una adquisición o modificación de una conducta (i.e. conocimiento, habilidad, actitud e información) o de su ejecución (Díaz, 2012). De hecho, la literatura muestra que cada persona posee un estilo característico en la forma de adquirir conocimientos.

En esta línea, Gallego y Martínez (2003) se refieren al

Fecha recepción: 07-04-20. Fecha de aceptación: 09-07-20 María Huertas González-Serrano

mh.gonzalez@ucv.es estilo de aprendizaje como el canal preferencial del alumno a la hora de captar la información, es decir, algunos estudiantes centran su atención en aspectos detallados mientras que otros lo hacen en aspectos lógicos; otros prefieren un mayor grado de independencia, otros prefieren estudiar junto a compañeros o profesores, a su vez unos prefieren leer y otros prefieren actividades prácticas. La teoría triárquica de Stenberg (1997) o la teoría de las inteligencias múltiples (Gardner, 2011), fundamentan la variabilidad de aptitudes frente al aprendizaje. Estas teorías fueron corroboradas posteriormente por la programación neurolingüística (Cudicio, 1996), la cual explica que cada persona posee un sistema sensorial dominante (visual, auditivo o kinésico) a través del cual su cerebro asimila mejor la información.

Por lo tanto, la variabilidad en el aprendizaje es un agente importante a tener en cuenta en la educación, pero existe otro constructo importante, el estilo de enseñanza. Como explican Mosston y Ashworth (1993), los estilos de enseñanza muestran cómo se desarrolla la interacción entre el docente y el alumno en el proceso de toma de decisiones, además de definir el rol de cada uno en este proceso. Romero y González (2001), explican los estilos de enseñanza como la forma peculiar de elaborar un programa docente y de diseñar y aplicar los métodos durante las actividades. Por lo tanto, el 
estilo de enseñanza hace referencia al cómputo de herramientas metodológicas empleadas por el docente para transmitir aptitudes y conocimientos al alumno de una manera eficaz, motivante y duradera (Canizales, Ries \& Rodríguez, 2020; Fernández Rivas \& Espada Mateos, 2017; Shen \& Chen, 2007). Existe una gran variedad de evidencia científica en lo que respecta a estilos de enseñanza y estilos de aprendizaje, abriendo un amplio abanico de posibilidades y matices, por lo que es importante que el docente conozca el estilo de aprendizaje de cada estudiante, y concretamente de cada grupo, para desarrollar un aprendizaje significativo (Alcívar, Ávila \& Reyes, 2019). Por ello, Chiang, Salgado, Diaz Larenas, Rivas Aguilera, y Martinez-Geijo (2013) realizaron una identificación y correlación de los estilos de enseñanza y de aprendizaje, determinando que el estilo activo de aprendizaje se correlacionaba con el estilo de enseñanza abierto, el reflexivo con el formal, el teórico con el estructurado y el estilo de aprendizaje pragmático con el estilo de enseñanza funcional.

Tomando como referencia a Renés et al. (2013), el estilo de enseñanza abierto o activo es aquel donde el docente promueve contenidos novedosos, motivando a los alumnos con diferentes actividades y en búsqueda de la originalidad. Suelen realizar dinámicas de trabajo en equipo y de participación activa del estudiante, variando en la metodología y quedando abierto a que los alumnos/as asuman diferentes roles. Siguiendo a estos mismos autores, el estilo de enseñanza formal o reflexivo es aquel donde el docente presenta una planificación detallada de los contenidos y se lo comunica a los alumnos. Suelen ceñirse a lo preestablecido y abordan las explicaciones con actividades detallas y analizando el contenido desde diferentes perspectivas. Fomentan en el alumno la reflexión y el análisis que sustenten ideas racionales, por lo que suelen promover el trabajo individual sobre el grupal. En este mismo trabajo, se señala que el estilo de enseñanza estructurado o teórico le otorga una gran importancia a la estructuración de la planificación y que los contenidos se sustenten en un amplio marco teórico. Suelen mantener una misma metodología y en un mismo ambiente de trabajo, favoreciendo agrupamientos homogéneos intelectualmente, valorando el proceso sobre la solución. Y, por último, el estilo de enseñanza funcional o pragmático es aquel usado por los docentes que ponen el énfasis en la viabilidad y la funcionalidad de la planificación. Los contenidos procedimentales tienen especial importancia y la dinámica de clase suele ser fluida y vivencial, siendo partidarios del trabajo en equipo.

Las investigaciones previas relacionadas con los estilos de enseñanza en la educación física (en adelante EF) ponen de manifiesto que los estilos de enseñanza más influyentes en el rendimiento académico son el mando directo y la asignación de tareas (Derri \& Pachta, 2007; Zeng, Leung, Liu, \& Bian, 2009). Aunque otros estilos como el descubrimiento guiado o la enseñanza recíproca influyen principalmente en el ambiente de clase, el nivel socio-afectivo de los alumnos o en la motivación que, de forma indirecta, también influyen en el rendimiento académico (Morgan, Kingston, \& Sproule, 2005; Salvara, Jess, Abbott, \& Bognár, 2006). Aun así, Isaza (2014) determinó que los profesores suelen adoptar estilos de enseñanza participativos, individuales, creativos, socializadores y cognitivos, tratando de evitar los estilos tradicionales. Normalmente, los profesores de EF utilizan una amplia variedad de estilos de enseñanza en sus clases (Syrmpas, Digelidis \& Watt, 2016), existiendo diferencias según la edad de estos (Jaakkola \& Watt, 2011; MerinoBarrero, Valero-Valenzuela \& Moreno-Murcia, 2017; Syrmpas \& Digelidis, 2014).

El estilo de enseñanza del docente y el estilo de aprendizaje del alumno conforman el proceso de enseñanza-aprendizaje. Pero los alumnos están fuertemente influenciados por el entorno y, en consecuencia, por sus familiares (Alcívar et al., 2019). Sin embargo, no existen estudios que analicen la perspectiva de padres y madres respecto a los diferentes estilos de enseñanza. Varios estudios, como el realizado por Gil y Contreras (2003), examinan la perspectiva de los padres en cuanto a la importancia de la asignatura, pero no se centran en el método de enseñanza de la propia asignatura. Por ello, los objetivos del presente estudio son: (1) conocer el estilo de aprendizaje preferido por los alumnos, el estilo predominante de enseñanza de los docentes de los alumnos de Educación Secundaria Obligatoria (ESO) y el estilo preferido de enseñanza por parte de los padres y madres de los alumnos; y por otro lado, (2) analizar qué factores se correlacionan e influyen en la existencia de diferencias estadísticamente significativas en las puntuaciones de los distintos estilos de enseñanza-aprendizaje de estos tres agentes educativos (alumnos/as, docentes y padres y madres).

\section{Material y método}

\section{Participantes}

La muestra está compuesta por un total de 198 estudiantes de secundaria, de los cuales el $48 \%$ chicas, y el 52\% eran chicos, con edades comprendidas entre los 12-16 años $(M=13.86 ; D T=1.32)$. En relación a los cursos, el $27 \%$ de estos alumnos eran de primero de la ESO, el $12 \%$ de segundo, el $30 \%$ de tercero y el $31 \%$ de cuarto curso. Asimismo, la muestra también está compuesta por 23 docentes que impartían clase a estos alumnos, y 104 padres y madres de dichos alumnos. Para la selección de la muestra se utilizó un muestreo no probabilístico o de conveniencia, y los datos fueron recogidos en las dos primeras semanas del mes de mayo de 2017.

\section{Instrumentos de evaluación}

En este estudio se utilizaron tres instrumentos distintos con la finalidad de recabar información de los tres estamentos que forman la comunidad educativa: (1) alumnos/as, (2) docentes y (3) padres y madres. En los tres casos, el instrumento utilizado fue un cuestionario auto reportado, cuyas características específicas se muestran a continuación.

Cuestionario estilos de aprendizaje para alumnos/as

El estilo de aprendizaje preferencial del alumno se evaluó mediante el cuestionario CHAEA-36 desarrollado por Alonso, Galledo, \& Honey (1999). Una adaptación del cuestionario estilos de aprendizaje para estudiantes de educación física (Maureira, 2015) compuesta por 36 ítems, 9 ítems para cada uno de los cuatro estilos de enseñanza-aprendizaje (activo, reflexivo, teórico y pragmático). El cuestionario se basa en una escala dicotómica donde 1 significa «Sí»» y 0 significa «No», y su valor final se obtiene mediante el sumatorio de 
todos los ítems, teniendo en cuenta las diferentes dimensiones del cuestionario.

Cuestionario estilos de enseñanza para profesores

El estilo de enseñanza preferencial de los profesores se evaluó mediante el cuestionario de Estilos de Enseñanza (CEE) desarrollado por Chiang Salgado et al. (2013). El cuestionario consta de 72 ítems, 18 de ellos dedicados a cada estilo de enseñanza: abierto, formal, estructurado y funcional y se basa en una escala dicotómica. Se basa en una escala dicotómica y, codificada tal y como se ha comentado en el anterior instrumento, atendiendo al sumatorio de los distintos ítems.

Cuestionario estilos de enseñanza para padres y madres

El estilo de enseñanza preferencial de los padres y madres se evaluó mediante un cuestionario de elaboración propia. Debido a la falta de cuestionarios validados que midan desde la perspectiva de enseñanza de padres y madres se procedió a elaborar un cuestionario propio de ocho ítems en base a los cuestionarios previamente utilizados en la investigación. Todas las preguntas de este cuestionario eran descriptivas, basadas en preguntas en las que los padres y madres tenían que elegir entre varias opciones de respuestas.

\section{Procedimiento}

En primer lugar, se habló con los órganos que dirigen el centro: directora y jefes de estudio para decidir cómo realizar los cuestionarios al alumnado. Todos los participantes recibieron una explicación detallada del protocolo de investigación, y tanto ellos como sus padres o tutores legales concedieron su consentimiento para la realización del estudio. Una vez obtenidos los permisos pertinentes, se subió el cuestionario a la plataforma Google Docs, de manera que todos los cuestionarios se realizaron online. El estudio fue diseñado de acuerdo con las pautas éticas de la Declaración de Helsinki de 1961 (World Medical Association, 2013).

Al final, y con la indispensable colaboración tanto de los profesores como de los alumnos, al principio de cada clase de Educación Física, los alumnos mediante sus teléfonos móviles realizaron la encuesta. En todo momento se informó a los alumnos/as del carácter voluntario para rellenar el cuestionario y se incidió también en el anonimato de los datos. En cuanto al tiempo de duración requerido para la contestación del cuestionario, éste fue de 12 minutos aproximadamente.

Finalmente, a los profesores se les hizo llegar el enlace de la encuesta a través de un correo electrónico de la directora. $\mathrm{Y}$ a los padres y madres también les llego en enlace a través de un correo electrónico enviado por el AMPA.

\section{Análisis estadístico}

Los datos de cada uno de los cuestionarios fueron codificaron en tres bases de datos diferentes, y estos fueron depurados antes de proceder a realizar los análisis estadísticos. En primer lugar, se procedió al cálculo de estadísticos descriptivos (frecuencias y porcentajes) además de medias y desviaciones típicas. En según lugar, se realizaron correlaciones para descubrir si existía algún tipo de asociación entre la preferencia o utilización de uno u otro tipo de estilo de enseñanza-aprendizaje y las puntuaciones de estos. En tercer lugar, se procedió a realizar comparaciones entre grupos.
En el caso de las variables cuantitativas, cuando las comparaciones eran entre dos grupos, se utilizó la prueba $t$ de Student para muestras independientes. Además, se calculó la $d$ de Cohen para comprobar el tamaño del efecto. Cuando las comparaciones eran entre más de dos grupos se realizó un análisis de ANOVA de un factor. Además, en aquellos casos en los que se encontró alguna diferencia significativa se realizó un contraste a posteriori mediante las pruebas posthoc de Bonferroni y Tamhane, dependiendo de los resultados arrojados respecto a la homogeneidad de las varianzas. También, para conocer el tamaño del efecto se calculó el eta al cuadrado ( $\eta 2$.$) .$

Finalmente, también se realizaron comparaciones entre grupos de variables cualitativas. En este caso, se utilizó la prueba del Chi-cuadrado de Pearson para conocer si existía algún tipo de relación entre estas variables $(p<.05)$. En este caso, se calculó el coeficiente de contingencia para comprobar el tamaño del efecto. Todos los análisis se realizaron utilizando el software IBM SPSS V. 22.0 (Armonk, NY: IBM Corp) y se estableció un nivel de significación de $p<.05$.

\section{Resultados}

A continuación, en este apartado se muestran todos los resultados relativos a los estilos de enseñanza-aprendizaje relativos en primer lugar a los alumnos/as, en segundo lugar, a los docentes, y en tercer lugar a los padres y madres de estos alumnos. Finalmente, se realiza una comparativa de las puntuaciones de los alumnos/as, docentes EF y padres/madres de estos alumnos encuestados.

\section{Resultados alumnos/as}

En primer lugar, se analizó si existía alguna relación entre los diferentes estilos de aprendizaje y el género, el curso de los estudiantes, las notas, el gusto por la EF y la práctica de actividad física extraescolar (ver Tabla 1). Los alumnos mostraron una relación positiva con el estilo teórico $(r=.15 ; p=.03)$. Además, el estilo teórico muestra una relación positiva con el gusto por la educación física $(\mathrm{r}=.17, p=.01)$, indicando que los alumnos con un estilo preferencial teórico muestran mayor predisposición por la materia. El estilo de enseñanzaaprendizaje activo muestra una relación inversa con el rendimiento académico y el gusto por la $\mathrm{EF}(r=-.20 ; p=.01 ; r=-0.15$; $p=.04$, respectivamente). Sin embargo, no se encontró ninguna relación de manera estadísticamente significativa respecto a los estilos de enseñanza-aprendizaje y el curso académico o la práctica de EF extraescolar.

\begin{tabular}{|c|c|c|c|c|}
\hline & Activo & Reflexivo & Teórico & Pragmático \\
\hline Género & -.07 & -.01 & $.16^{*}$ & .09 \\
\hline Curso ESO & .11 & -.10 & -.07 & -.08 \\
\hline Nota EF & $-.20^{* *}$ & -.20 & .82 & -.90 \\
\hline Gusto EF & $-.15^{*}$ & .36 & $.17^{* *}$ & -.28 \\
\hline Práctica EF extraescolar & .22 & .17 & .10 & -.08 \\
\hline
\end{tabular}

A continuación, se procedió a analizar si existían diferencias estadísticamente entre el género y los distintos estilos de enseñanza-aprendizaje. Como se puede observar en la Tabla 2, los chicos presentaron mayores medias en el caso del estilo de aprendizaje teórico $(M=6.51, D T= \pm 1.69)$, mientras que las chicas lo hicieron en el caso del aprendizaje 
reflexivo $(M=6.04, D T= \pm 1.70)$. Sin embargo, estas diferencias únicamente fueron significativas en el caso del estilo de enseñanza-aprendizaje teórico, donde fueron los chicos los que presentaron medias significativamente mayores que las de las chicas $(p=.03)$.

Tabla 2

\begin{tabular}{lccccc}
\multicolumn{7}{l}{ Características descriptivas de los alumnos del estudio } & & & \\
\hline & Todos & Chicos & Chicas & $p$ & $\begin{array}{c}\text { dde } \\
(M, D T)\end{array}$ \\
$(M, D T)$ & $(M, D T)$ & $p o h e n$ \\
\hline Activo & $5.70 \pm 1.91$ & $5.58 \pm 2.08$ & $5.83 \pm 7.72$ & .58 & - \\
Reflexivo & $6.04 \pm 1.90$ & $6.04 \pm 2.07$ & $6.04 \pm 1.70$ & .32 & - \\
Teórico & $6.24 \pm 1.79$ & $6.51 \pm 1.69$ & $5.94 \pm 1.84$ &. .03 & .17 \\
Pragmático & $5.75 \pm 1.75$ & $5.88 \pm 1.56$ & $5.60 \pm 1.51$ & .51 & - \\
\hline
\end{tabular}

Se analizaron las diferencias en las puntuaciones entre los distintos estilos de enseñanza aprendizaje entre los alumnos/as según su nota en EF (ver Tabla 3). Los alumnos que obtuvieron un insuficiente presentaron puntuaciones más altas en el estilo activo, mientras que los alumnos que obtuvieron un suficiente y calificaciones mayores, presentaron las puntuaciones más altas en el estilo teórico. Se encontraron diferencias estadísticamente significativas según las notas de los alumnos en EF en el estilo activo $(p=.03)$. Concretamente, estas diferencias estadísticamente significativas $(p=.02)$ se encontraron entre los alumnos que presentaron un insuficiente $(M=7.57, D T= \pm 1.40)$ en la materia los alumnos/as que obtuvieron sobresaliente $(M=5.10, D T= \pm 11.59)$, siendo las medias más elevadas en el caso de estos primeros.

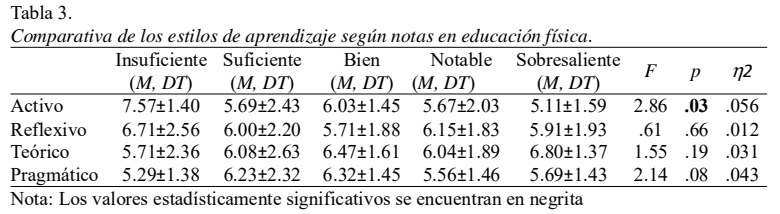

Por otra parte, en relación al gusto por la educación física y los distintos estilos de enseñanza-aprendizaje. El estilo pragmático, activo y reflexivo están asociados con los alumnos que no les gustaba nada la clase de EF $(M=7.50$, $D T= \pm 2.12 ; M=6.50, D T= \pm 2.12 ; M=6.50 \pm 0.77$, respectivamente). El estilo teórico está asociado con los alumnos que les gusta mucho la clase de EF ( $M=6.73, D T= \pm 1.66)$. Además, se encontraron diferencias estadísticamente significativas entre el estilo teórico de los alumnos/as que no le gustaban nada la EF y entre aquellos que les gustaba mucho $(F=3.32$; $p=.01)$. A continuación, en la tabla 4 se muestran los resultados:

\begin{tabular}{|c|c|c|c|c|c|c|c|c|}
\hline & $\begin{array}{c}\text { Nada } \\
(M, D T)\end{array}$ & $\begin{array}{c}\text { Poco } \\
(M, D T)\end{array}$ & $\begin{array}{l}\text { Más o menos } \\
\quad(M, D T)\end{array}$ & $\begin{array}{l}\text { Bastante } \\
(M, D T)\end{array}$ & $\begin{array}{l}\text { Mucho } \\
(M, D T)\end{array}$ & $F$ & $p$ & $\eta 2$ \\
\hline Activo & $6.50 \pm 2.12$ & $5.33 \pm 2.10$ & $6.27 \pm 1.90$ & $5.82 \pm 2.04$ & $5.24 \pm 1.64$ & 2.29 & .61 & .045 \\
\hline Reflexivo & $6.50 \pm 0.77$ & $6.17 \pm 1.70$ & $5.71 \pm 2.03$ & $6.01 \pm 1.72$ & $6.25 \pm 1.05$ & .59 & .67 & .012 \\
\hline Teórico & $3.50 \pm 2.12$ & $6.50 \pm 1.31$ & $5.93 \pm 2.05$ & $6.00 \pm 1.67$ & $6.73 \pm 1.66$ & 3.32 & .01 & .064 \\
\hline Pragmático & $7.50 \pm 2.12$ & $6.33 \pm 1.56$ & $5.38 \pm 1.75$ & $5.83 \pm 1.51$ & $5.75 \pm 1.35$ & 1.82 & .13 & .036 \\
\hline
\end{tabular}

Finalmente, también se compararon si existían diferencias estadísticamente significativas entre la preferencia de los distintos estilos de aprendizaje según el curso de la ESO en el que se encontraban los alumnos y si practicaba actividad física de manera extraescolar. Sin embargo, no se encontraron diferencias estadísticamente significativas $(p>.05)$.

\section{Resultados docentes}

Por otra parte, se procedió a analizar si existían relaciones estadísticamente significativas entre las características de los docentes (experiencia laboral y edad), y la utilización de uno u otro estilo de enseñanza-aprendizaje. Como se puede observar en la Tabla 5, no se encontraron relaciones de manera estadísticamente significativas entre los años de experiencia docente y los diferentes estilos de enseñanza-aprendizaje. Solamente se encontró una correlación estadísticamente significativa y en sentido positivo entre la edad y el estilo estructurado $(r=.50 ; p=.03)$, y entre la edad y el estilo abierto ( $r=-.48 ; p=.04)$, siendo la relación en este caso negativa.

Tabla 5.

Correlaciones entre los diferentes estilos de enseñanza-aprendizaje y las variables nota y gusto por la educación fisica.

\begin{tabular}{|c|c|c|c|c|}
\hline & Abierto & Formal & Estructurado & Funcional \\
\hline Experiencia docente & -.35 & -.16 & .41 & -.12 \\
\hline Edad & $-.48^{*}$ & .15 & $.50^{*}$ & -.68 \\
\hline
\end{tabular}

Posteriormente, se procedió a analizar si existían diferencias estadísticamente significativas entre la edad del docente y las distintas puntuaciones de los estilos de enseñanzaaprendizaje. Los docentes más jóvenes (de 25 a 44 años) y los de mediana edad (35-45 años), presentaron mayores medias en el estilo abierto $(M=14.33, D T= \pm 1.15 ; M=13.43$, $D T= \pm 1.99$, respectivamente), mientras que los docentes de 45-54 años lo hicieron en el estilo estructurado ( $M=12.40$, $D T= \pm 1.51$ ). Finalmente, los docentes de mayor edad presentaron mayores medias en el estilo formal $(M=13.50 \pm 2.12)$. Sin embargo, solamente se encontraron diferencias de manera estadísticamente significativa en el estilo estructurado entre los docentes de 55-65 años y los docentes de 45-54 años, con los dos otros grupos de docentes más jóvenes $(p<.05)$, presentando medias más altas aquellos profesores de mayor edad. Además, también se encontraron diferencias estadísticamente significativas en el estilo abierto entre los docentes de menor edad (25-34 años) y aquellos de una edad media y avanzada (45 años o más). A continuación, en la Tabla 6 se pueden observar los resultados.

\begin{tabular}{|c|c|c|c|c|c|c|c|}
\hline \\
\hline & $\begin{array}{c}25-34 \text { años } \\
(M, D T)\end{array}$ & $\begin{array}{c}\text { 35-44 años } \\
(M, D T)\end{array}$ & $\begin{array}{c}\text { 45-54 años } \\
(M, D T)\end{array}$ & $\begin{array}{c}\text { 55-65 años } \\
(M, D T)\end{array}$ & $F$ & $p$ & $\eta 2$. \\
\hline Abierto & $14.33 \pm 1.15$ & $13.43 \pm 1.99$ & $11.80 \pm 2.10$ & $12.72 \pm 2.07$ & 1.69 & .02 & .220 \\
\hline Formal & $10.33 \pm 3.10$ & $10.43 \pm 2.94$ & $11.70 \pm 2.11$ & $13.50 \pm 2.12$ & 1.01 & 0.41 & .144 \\
\hline Estructurado & $9.67 \pm 2.08$ & $10.43 \pm 2.44$ & $12.40 \pm 1.51$ & $13.00 \pm 1.41$ & 2.76 & .04 & .315 \\
\hline Funcional & $12.33 \pm 0.58$ & $11.14 \pm 1.86$ & $11.80 \pm 2.20$ & $12.50 \pm 2.12$ & .41 & .75 & .064 \\
\hline
\end{tabular}

Finalmente, se analizó si existían diferencias entre los diferentes grupos de docentes según su experiencia laboral, pero no se encontró ninguna diferencia de manera estadísticamente significativa en relación a los estilos de enseñanza-aprendizaje ( $p>.05)$.

\section{Resultados padres y madres}

A continuación, se analizaron los datos referentes a los padres y madres de estos alumnos/as. En primer lugar, es necesario destacar que la satisfacción de los padres y madres respecto al profesorado es alta. En concreto, el profesorado el $75.50 \%$ contestaron que están bastante satisfechos de los profesores/as de sus hijos, seguido de un $13.70 \%$ que están muy satisfechos con el profesorado.

Posteriormente, se analizó las relaciones entre las opiniones que tienen los padres sobre la educación de sus hijos, y la preferencia por los diferentes estilos de enseñanzaaprendizaje (ver tabla 7). En relación a la primera pregunta sobre qué es lo importante en el instituto para estos padres y madres, la mayoría de ellos creen que lo importante en el 
instituto «vivenciar cambios y diferentes situaciones para aprender a afrontarlos» (42.20\%), seguido de «el desarrollo social y la convivencia» con un $22.50 \%$, y de «desarrollar diferentes estrategias para solucionar incógnitas» con un $20.80 \%$ de los padres y madres contestando estas opciones. En cuanto a los resultados de la prueba del Chi-cuadrado, este resultó ser no significativo $\left(x^{2}=15.03, g l=12, p=.24\right)$. Por lo tanto, no existe ningún tipo de asociación entre lo que los padres/madres consideran importante en el instituto, y las preferencias por los diferentes estilos de enseñanza-aprendizaje.

En relación a la pregunta sobre las notas de sus hijos/as, el $75.50 \%$ de los padres y madres contestaron que «si estudia y se esfuerza no me importan las notas». En segundo lugar, un $17.60 \%$ contestó que «exijo que saque notables y sobresalientes», y en tercer lugar el $6.90 \%$ restante contesto que «me conforma con lo que saque su/s hijo/s». Por lo que respecta a los resultados de la prueba del Chi-cuadrado, este también resultó ser no significativo $\left(x^{2}=8.61, g l=6, p=.20\right)$. Por lo tanto, tampoco existe ningún tipo de asociación entre lo que les exigen los padres y madres a sus hijos/as en las notas, y la preferencia por uno u otro estilo de enseñanzaaprendizaje.

Respecto a la pregunta cuándo es bueno un profesor, un $47.10 \%$ contestaron que es aquel que «invierte tiempo con los alumnos en reflexionar». Un porcentaje similar de padres/ madres $(47.10 \%)$, contestó que un buen profesor es aquel que «enseña diferentes caminos para que el/la alumno/a busque la solución». En cuanto a los resultados de la prueba del chi-cuadrado, este resultó ser no significativo $\left(x^{2}=6.29\right.$, $g l=9, p=.71)$. Por lo tanto, no existe ningún tipo de asociación entre la percepción de cuándo un profesor es bueno, y la preferencia por uno u otro estilo de enseñanza-aprendizaje.

Seguidamente, por lo que respecta a la preferencia de los padres y madres durante las clases de su hijos/as, el 93.10\% contestó que prefieren que tanto el centro como los profesores innoven en la educación, frente a un 6.90\% que contestó que prefieren que se den las clases de toda la vida. En cuanto a los resultados de la prueba del chi-cuadrado, este resultó ser significativo $\left(x^{2}=17.29, g l=3, p=.001\right)$. Concretamente, se encontró una relación entre los padres y madres que prefieren que tanto el centro como los profesores innoven en la educación, y la preferencia por los estilos de enseñanza activo, pragmático y reflexivo.

Finalmente, en cuanto a la preferencia de los padres y madres dentro del aula, el 77.70\% defiende que lo más importantes es que «se razone y se debata durante las clases», mientras que un $18.40 \%$ opina que lo más importante es «que se trabajar en equipo con los compañeros». En cuanto a los resultados de la prueba del chi-cuadrado, este resultó ser significativo $\left(x^{2}=31.39, g l=9, p=.000\right)$. Concretamente, se encontró una relación entre los padres y madres que consideran como lo más importante en el aula que se razone y se debata, y la preferencia por los estilos de enseñanza reflexivo y activo. A continuación, en la tabla 7 se pueden observar los resultados:

\section{Comparativa resultados preferencias metodologías:} alumnos, padres y profesores

Por último, con la finalidad de cotejar los resultados de todos los agentes que intervienen en el proceso de enseñanza-aprendizaje durante la formación del alumno, finalmente se compararon los diferentes resultados obtenidos entre alumnado, profesorado y familiares (ver Figura 1). A partir de los datos, se demuestra que no existe ninguna concordancia entre la preferencia de estilos de los tres agentes de la comunidad educativa, ya que el estilo con una mayor puntuación entre los alumnos/as es el teórico con un promedio de 6.24, a su vez, el estilo predominante entre los profesores/as de EF es el activo con una media de 14.10, y, por último, tanto los padres como las madres prefieren un estilo reflexivo (49\%). En la siguiente Figura 1 se pueden observar los resultados.

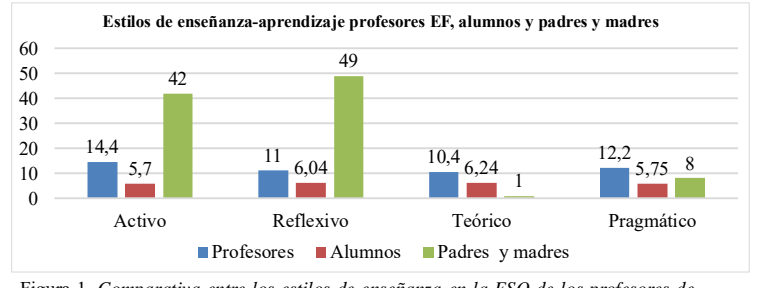

Figura 1. Comparativa entre los estilos de enseñanza en la ESO de los profesores de educación física, los alumnos y los padres y madres.

Tabla 7.

Distribución porcentual, total, ??2 y p valor según entienden los padres/madres la educación de sus hijos/as de acuerdo a la preferencia de los estilos de aprendizaje según padres y madres. Estilos de enseñanza-aprendizaje Lo importante en el instituto es..

Las notas

El desarrollo social y la convivencia

Vivenciar cambios y diferentes situaciones para aprender a afrontarlos Desarrollar diferentes estrategias para solucionar incógnitas Otros Total (\%)

En cuanto a las notas de mis hijos..

Le exijo que saque notables y sobresalientes

Me conformo con lo que saque

Si estudia y se esfuerza no me importan las notas

Total (\%)

Un profesor es bueno cuando...

Mi hijo sabe la solución al final de la clase

Enseña diferentes caminos para que el alumno/as busque la solución

Invierte tiempo en reflexionar con los alumnos/as

Es claro y conciso

Total (\%)

Prefiero que...

Se den las clases de toda la vida

Tanto el centro como los profesores innoven en la educación

Total (\%)

En un aula, lo más importante es...

Planificar

Que se trabaje en equipo con los compañeros

Que se razone y se debata

El trabajo individualizado

Total

Nota: T-Teórico; R-Reflexivo; A- Activo; P-pragmático; res-residuo tipificado; Cof. Cont-coeficiente de contingencia.

\begin{tabular}{|c|c|c|c|c|c|c|}
\hline$T$ & $\mathbf{R}$ & $\mathbf{A}$ & $\mathbf{P}$ & $x^{2}$ & $p$ & Coef. Cont. \\
\hline$\%$ (res) & $\%$ (res) & $\%$ (res) & $\%$ (res) & & & \\
\hline $0(-.30)$ & $2.00(-1.20)$ & $7.10(1.70)$ & $0(-.90)$ & & & \\
\hline $1(1.60)$ & $12.20(.30)$ & $8.20(-.30)$ & $1(-.60)$ & & & \\
\hline $0(-.70)$ & $26.50(1.10)$ & $13.30(-1.00)$ & $3.10(-.20)$ & & & \\
\hline $0(-.30)$ & $3.10(-.90)$ & $5.10(.50)$ & $2(2.00)$ & 15.03 & .24 & - \\
\hline $0(-.40)$ & $6.10(-.50)$ & $7.10(.40)$ & $2(.70)$ & & & \\
\hline 1 & 50 & 40.80 & 8.20 & & & \\
\hline$\%$ (res) & $\%$ (res) & $\%$ (res) & $\%$ (res) & $x^{2}$ & $p$ & Coef. Cont. \\
\hline $1(2.00)$ & $5.10(-1.20)$ & $10.10(1.10)$ & $1.00(-.30)$ & & & \\
\hline $0(-.30)$ & $4.00(.30)$ & $2.00(-.50)$ & $1.00(.60)$ & & & \\
\hline $0(-.90)$ & $40.40(.50)$ & $29.30(-.40$ & $6.10(0)$ & 8.61 & .20 & - \\
\hline 1 & 49.50 & 41.40 & 8.10 & & & \\
\hline$\%$ (res) & $\%$ (res) & $\%$ (res) & $\%$ (res) & $x^{2}$ & $p$ & Coef. Cont. \\
\hline $0(-.10)$ & $1.00(-.70)$ & $0(-.60)$ & $0(-.30)$ & & & \\
\hline $0(-.70)$ & $19.00(-.70)$ & $24(1.19)$ & $3.00(-.40)$ & & & \\
\hline $1.00(.80)$ & $26.00(.50)$ & $17.00(-.70)$ & $4.00(.10)$ & 6.29 & .71 & - \\
\hline $0(-.20)$ & $3.00(.40)$ & $1.00(-.80)$ & $1.00(.90)$ & & & \\
\hline 1 & 49 & 42 & 8 & & & \\
\hline$\%$ (res) & $\%$ (res) & $\%$ (res) & $\%$ (res) & $x^{2}$ & $p$ & Coef. Cont. \\
\hline $1.00(3.50)$ & $1.00(-1.30)$ & $5.00(1.20)$ & $0(-.70)$ & & & \\
\hline $0(-1.00)$ & $48.00(.40)$ & $37.00(-3.00)$ & $8(.20)$ & 17.29 & .001 & $.384(p=.001)$ \\
\hline 1 & 49 & 42 & 8 & & & \\
\hline$\%$ (res) & $\%$ (res) & $\%$ (res) & $\%$ (res) & $x^{2}$ & $p$ & Coef. Cont. \\
\hline $1.00(4.80)$ & $1.00(-.70)$ & $2.00(.20)$ & $0(-.60)$ & & & \\
\hline $0(-.40)$ & $7.00(-.60)$ & $9.00(.50)$ & $2.00(.50)$ & & & \\
\hline $0(-.90)$ & $40(.50)$ & $31(-.20)$ & $5.00(-.40)$ & 31.39 & .000 & $.489(p=.001)$ \\
\hline $0(-.10)$ & $1.00(.00)$ & $0(-.90)$ & $1(2.10)$ & & & \\
\hline 1 & 49 & 42 & 8 & & & \\
\hline
\end{tabular}




\section{Discusión}

El objetivo principal del estudio fue conocer cuáles son los estilos de enseñanza-aprendizaje preferidos por los alumnos/as, cuales utilizan sus docentes, y cuáles prefieren sus padres, así como analizar los factores que influyen en esta elección. Esta investigación resulta de interés puesto que uno de los grandes retos de los profesores comprometidos con la mejora continuo de su quehacer pedagógico, es qué rol adoptar para desarrollo de la capacidad de aprendizaje del alumno (Canizales, Ries \& Rodríguez, 2020). Los resultados señalan una discordancia en el proceso de enseñanza-aprendizaje entre los diferentes agentes educativos. Los alumnos prefieren el estilo teórico, los docentes suelen emplear un estilo abierto y los familiares están más de acuerdo con un estilo reflexivo. Estos resultados ponen de manifiesto la distancia entre las políticas formativas y las expectativas del alumnado en las clases de EF (Tobar, Freundt, Gaete, \& Lara, 2020). Además, se encontraron que ciertos factores se correlacionan e influían en la elección de los estilos de enseñanza-aprendizaje de estos tres agentes educativos (alumnos/as, docentes y padres y madres).

En primer lugar, respecto al estilo de aprendizaje preferido por el alumnado, se observaron diferencias entre chicos y chicas, los chicos prefieren un estilo más teórico mientras que las chicas prefieren el estilo reflexivo. En contraposición a nuestro estudio, Gil et al. (2007) determina que el estilo preferencial de todos los alumnos es el reflexivo. Nuestros resultados reflejan que los alumnos más cercanos al estilo teórico eran más afines a la clase de educación física, y obtuvieron calificaciones más altas en esta materia. Otros estudios como el realizado por Pastor et al. (2006), muestran como estilos de enseñanza-aprendizaje más activos y participativos en la clase de educación física mejoraron la implicación del alumnado y su rendimiento académico. Esta discordancia entre la literatura previa y nuestros resultados puede deberse a que los participantes de nuestro estudio se hayan visto influenciados por métodos más tradicionales durante su escolarización, y ello les conlleve a sentirse más cómodos en el estilo teórico.

En segundo lugar, centrándonos en los docentes, la literatura actual muestra como los profesores/as se han centrado en desarrollar y ampliar sus conocimientos respecto a los contenidos curriculares y los conocimientos pedagógicos del contenido (Castejón \& Giménez, 2017; Monique \& Malini 2010), pero poco se sabe sobre la adaptación del docente en cuanto al estilo de aprendizaje del alumnado. Nuestros resultados concuerdan con el estudio realizado por DelgadoNoguera (2015), que afirma que durante la didáctica actual se suelen utilizar variedad de estilos de enseñanza-aprendizaje durante el proceso de formación del alumnado. Los valores obtenidos en los cuatro estilos de enseñanza-aprendizaje son muy próximos entre sí, reafirmando la variabilidad de estilos de los docentes. Además, nuestro estudio encontró diferencias en el estilo de enseñanza del profesorado teniendo en cuenta la edad de los docentes, lo que se encuentra en línea con estudios previos (Syrmpas \& Digelidis, 2014; Merino-Barrera et al., 2017). En este caso, los más jóvenes se mostraron afines al estilo activo, mientras que los profesores de más edad se mostraron más cercanos al estilo estructura- do o teórico. Las diferencias entre los estilos de enseñanza respecto a la edad de los profesores podrían explicar la variabilidad de estilos docentes en el centro. El contexto docente está evolucionando y pasando de estilos tradicionales y teóricos a estilos más activos y reflexivos (Rodríguez-Fernández, Mato-Cadórniga, \& Pereira-Mariño, 2016). Esto puede ser debido a que cuando los docentes utilizan estilos de enseñanza que permiten que los alumnos participen y estén implicados en el diseño de tareas y en la toma de decisiones, se generan unos mayores niveles disfrute de la práctica física (Zueck, García, Villalobos \& Gutiérrez, 2020). Sin embargo, en función de la experiencia laboral no se encontraron diferencias estadísticamente significativas, lo que se encuentra en línea con estudios previos (Jaakkola \& Watt, 2011).

En cuanto a la perspectiva de las familias dentro del proceso de enseñanza-aprendizaje, estudios como el realizado por Oliva, Parra y Arranz (2008), determinan estilos padrehijo/a y madre-hijo/a y los enmarcan como modelos democráticos, estrictos o indiferentes. Estos modelos pueden favorecer el estilo preferencial de aprendizaje de sus hijos/as hacia un modelo u otro, pero no hemos encontrado estudios que tengan en cuenta los estilos de enseñanza-aprendizaje preferencial de los familiares de los alumnos. Nuestros resultados muestran que los familiares presentan una alta disposición a que se innove en el aula y se utilicen estilos de enseñanza activos y reflexivos. Además, se encontraron relaciones significativas entre los padres y madres que prefieren que tanto el centro como los profesores innoven en la educación, y que consideran como lo más importante en el aula que se razone y se debata, y la preferencia por los estilos de enseñanza activo, pragmático y reflexivo.

Estudios previos como el de Rios y Maldonado (2017) afirman que los estilos de aprendizaje están relacionados con las teorías de la enseñanza y que los alumnos son capaces de adaptarse, pero a pesar de las posibles adaptaciones, la realidad es que no todos los alumnos aprenden desde un mismo método. Alcívar et al. (2019), en cambio, habla sobre la necesidad de que el docente adapte los métodos de enseñanza a sus alumnos, con el fin de conectar con ellos y conseguir despertar su interés. Además, la figura de los familiares debería tenerse en cuenta ya que podría influir de forma directa en el proceso, facilitando la labor del docente sin amenazar su figura de autoridad en el aula (Navarro, Pérez, González, Mora, \& Jiménez, 2006). Por lo tanto, las competencias docentes son un factor primordial en las clases de EF para crear un clima de aprendizaje que repercuta positivamente en el comportamiento y aprendizaje de los alumnos/as (Baños \& Arrayales, 2020)

\section{Conclusiones e implicaciones prácticas}

No existe concordancia entre los estilos de enseñanzaaprendizaje de los tres agentes de la comunidad educativa. El estilo con una mayor puntuación entre los alumnos/as es el teórico, el estilo predominante entre los docentes es el activo, y en los padres y madres es el reflexivo. En concreto, los alumnos muestran más preferencia por el estilo teórico que las alumnas, así como aquellos alumnos/as a los que le gusta mucho la EF. Sin embargo, los alumnos/as que suspendieron esta materia, prefieren un estilo activo. 
En relación a los profesores, se encontró que los profesores de menor edad prefieren un estilo activo, y los de mayor edad un estilo estructurado. Sin embargo, no se encontraron diferencias de acuerdo a la experiencia laboral. Por último, respecto a los padres y madre de los alumnos, se encontró relación entre aquellos que prefieren que se innove en la educación, y la preferencia por los estilos de enseñanza activo, pragmático y reflexivo. También se encontró una relación entre aquellos padres y madres que consideran que lo más importante en el aula que se razone y se debata en el aula, y la preferencia por los estilos de enseñanza reflexivo y activo.

Por lo tanto, el estudio de los estilos de enseñanza-aprendizaje de esta investigación puede ayudar a entender la realidad educativa de los centros, determinando qué estilos de aprendizaje prefieren los alumnos, cuales son los estilos de enseñanza más extendidos entre el personal docente, y cuál es la perspectiva familiar. Una concordancia entre todos los agentes educativos facilitaría la adquisición de conocimientos de los alumnos, la labor del docente y el apoyo aportado por los familiares. Es por ello, que los políticos, las instituciones educativas y los centros educativos deberían atender a los resultados de este estudio para poner en práctica técnicas que acerquen los estilos de enseñanza y aprendizaje entre todos los agentes educativos con el fin de mejorar el rendimiento académico de los alumnos de educación secundaria obligatoria. Además, de esta manera, se podría también conseguir una mayor adherencia a la práctica físico-deportiva a través de la materia de EF.

\section{Limitaciones y futuras líneas de estudio}

Finalmente, es necesario señalar que este estudio presenta una serie de limitaciones. La primera de ellas, es que la recogida de datos se realizó solamente en un centro de secundaria, por lo que los resultados no son extrapolables a toda la población de estudiantes se secundaria. Por lo tanto, futuros estudios deben de replicar este estudio con otras muestras de estudiantes, para ver si los resultados son generalizables. Asimismo, también sería interesante realizar un estudio con los mismos alumnos (longitudinal) determinando su estilo de aprendizaje durante las diferentes etapas de la educación. La segunda de ellas, es que el cuestionario que se les administró a los padres no estaba validado. Y eso conllevó a que no se utilizara la misma escala de medida entre los datos obtenidos mediante los cuestionarios validados (alumnos/as y profesores) y los obtenidos por el cuestionario de elaboración propia (padres y madres). Por lo tanto, como futura línea de estudio, si se quiere estudiar y profundizar sobre la enseñanza individualizada y los factores que le afectan, se deberían desarrollar y validar cuestionarios para padres, madres o familiares.

\section{Referencias}

Alcívar E. J., Ávila, F. M., \& Reyes, O. B. (2019). Modelos de estilos de aprendizaje, caso de estudio: Unidades Educativas Fiscales de Chone. Pro Sciences, 3(23), 31-37.

Alonso, C., Galledo, D., \& Honey, P. (1999). Los estilos de aprendizaje (4th ed.). Bilbao: Ediciones mensajero.
Baños, R., \&Arrayales, E. (2020). Predicción del aburrimiento en la educación física a partir del clima motivacional. Retos, 38, 83-88.

Berg, C. A., \& Stenberg, R. J. (1985). A triarchic theory of intellectual development during adulthood. Developmental Review, 5, 334-370.

Canizales, W., Ries, F., \& Rodríguez, C. (2020). Estilos de aprendizaje y ambiente de aula: situaciones que anteceden a la innovación pedagógica en estudiantes de deporte. Retos, 38(38), 213-221.

Castejon J., \& Giménez, F. J. (2017). Conocimiento del contenido y conocimiento pedagógico del contenido de educación física en educación secundaria. Retos, 32, 146151.

Chiang Salgado, M., Diaz Larenas, C., Rivas Aguilera, A., \& Martinez-Geijo, P. (2013). Validación del cuestionario Estilos de Enseñanza (CEE). Un instrumento para el docente de educación superior. Revista Estilos de Aprendizaje, 11(12), 1-15.

Cudicio, C. (1996). Comprender la PnI: la Programación Neurolingüistica, Herramienta de la Comunicación. (2nd ed.). Barcelona: Ediciones Granica SA.

Delgado Noguera, M. A. (2015). Los estilos de enseñanza de la Educación Física y el Deporte a través de 40 años de vida profesional. Federación Española de Asociaciones de Docentes de Educación Física, 2(2), 21-84.

Derri, V., \& Pachta, M. (2007). Motor skills and concepts acquisition and retention: a comparison between two styles of teaching. International Journal of Sport Science, 63(1), 37-47.

Díaz, E. (2012). Estilos de aprendizaje. Eidos, 5, 5-11.

Fernández Rivas, M., \& Espada Mateos, M. (2017). Formación inicial y percepción del profesorado sobre los estilos de enseñanza en Educación Física. Retos, 2041(31), 69-75.

Gallego,A., \& Martínez, E. (2003). Estilos deAprendizaje y ELearning. Hacia un mayor rendimiento académico. Revista de Educación a Distancia, 7, 1-36.

Gardner, H. (2011). Inteligencias múltiples: la teoría en la práctica (5th ed.). Barcelona: Paidos.

Gil, P., \& Contreras, O. R. (2003). Interés y valoración del área de educación física por padres y madres en la enseñanza obligatoria. Revista de Educación, 332, 327-355.

Gil, P., Contreras, O. R., Pastor, J. C., Gómez, I., González, S., García, L. M., \& López, A. (2007). Estilos de aprendizaje de los estudiantes de magisterio: Especial consideración de los alumnos de educación física. Revista de Currículum y Formación Del Profesorado, 11(2), 8-46.

Isaza, L. (2014). Estilos de aprendizaje: una apuesta por el desempeño académico de los estudiantes en la educación física. Revista Encuentros, 12(2), 25-34.

Jaakkola, T., \& Watt, A. (2011). Finnish physical education teachers' self-reported useand perceptions of Mosston and Ashworth's teaching styles. Journal of Teaching in Physical Education, 30, 248-262.

Maureira, F. (2015). CHAEA-36/ : Adaptación del Cuestionario Honey-Alonso de Estilos de Aprendizaje para estudiantes de Educación Física de Chile. Revista Electrónica de Psicología Iztacala, 18(3), 1133-1152.

Merino-Barrero, J. A., Valero-Valenzuela, A., \& Moreno-Mur- 
cia, J. A. (2017). Análisis psicométrico del cuestionario estilos de enseñanza en educación física (EEEF). International Journal of Medicine and Science of Physical Activity and Sport, 17(66), 225-241

Monique, L., \& Malini, R. (2010). Content knowledge in teaching, an investigation into an adequate 'milieu' for teaching dance: The case of Indian dance in France. European Physical Education Review, 16(1), 65-79.

Morgan, K., Kingston, K., \& Sproule, J. (2005). Effects of different teaching styles on the teacher behaviours that influence motivational climate and pupuls' motivation in physical education. European Physical Education Review, 11(3), 257-285.

Mosston, M., \& Ashworth, S. (1993). La Reforma de los Estilos de Enseñanza. Barcelona: Editorial Hispano Europea, S.A.

Navarro, G., Pérez, C., González, A., Mora, O., \& Jiménez, J. (2006). Revista Interamericana de Psicología. Interamerican Journal of Psychology, 40(2), 205-212.

OCDE (2017), Marco de Evaluación y de Análisis de PISA para el Desarrollo : Lectura, matemáticas y ciencias, Versión preliminar, OECD Publishing, Pari.

Oliva, A., Parra, Á., \& Arranz, E. (2008). Estilos relacionales parentales y ajuste adolescente. Infancia y Aprendizaje, 31(1), 93-106.

Pastor, V., Monjas, R., Gómez, J., López, E. M., Martín, J. F., González, J., ... Marugán, L. (2006). La evaluación en educación física. Revisión de modelos tradicionales y planteamiento de una alternativa. Retos, 10, 31-41.

Renés Arellano, P., Echeverry, L., Chiang Salgado, M., Rangel, L., \& Martínez Geijo, P. (2013). Estilos de enseñanza: un paso adelante en su conceptualización y diagnóstico. Revista de Estilos de Aprendizaje, 11(11), 4-18.

Rios, R. P., \& Maldonado, E. M. (2017). Relación entre los estilos de aprendizaje y las teorías de enseñanza. Retos, 32, 7-13.

Rodríguez Fernández, J. E., Mato Cadórniga, J. Á., \& Pereira Mariño, M. C. (2016). Análisis de los métodos tradiciona- les de enseñanza-aprendizaje de los deportes colectivos en Educación Primaria y propuestas didácticas innovadoras. Sportis, 2(2), 303-323.

Romero, J. F., \& González, M. J. (2001). Prácticas de comprensión lectora. Estrategias para el aprendizaje. Madrid: Alianza Editorial.

Salvara, M., Jess, M., Abbott, A., \& Bognár, J. (2006). A preliminaty study to investigate the influence of different teaching styles on pupils' goal orientations in physical education. European Physical Education Review, 12(1), 51-74.

Shen, B., \& Chen, A. (2007). An examination of learning profiles in physical education. Journal of Teaching in Physical Education, 2(26), 145-160.

Syrmpas, I., \& Digelidis, N. (2014). Physical education student teachers' experiences with and perceptions of teaching styles. Journal of Physical Education and Sport, 14(1), 52-59.

Syrmpas, I., Digelidis, N., \& Watt, A. (2016). An examination of Greek physical educators' implementation and perceptions of Spectrum teaching styles. European Physical Education Review, 22(2), 201-214.

Tobar, B. U., Freundt, A. M. R., Gaete, M. J. F., \& Lara, M. M. (2020). Paradigma educativo y habilidades del profesor asociadas a la percepción de rol docente en Educación Física de estudiantes chilenos. Retos, 37, 362-369.

World Medical Association. (2013). World Medical Association Declaration of Helsinki: ethical principles for medical research involving human subjects JAMA, 310,2191-2194.

Zeng, H. Z., Leung, R. W., Liu, W., \& Bian, W. (2009). Learning outcomes tought by three teaching styles in college fundamental voleyball classes. Clinical Kinesiology, 63(1), $1-6$.

Zueck, M. D. C., García, A. A. R., Villalobos, J. M. R., \& Gutiérrez, H. E. I. (2020). Satisfacción en las clases de Educación Física y la intencionalidad de ser activo en niños del nivel de primaria. Retos, 37, 33-40.

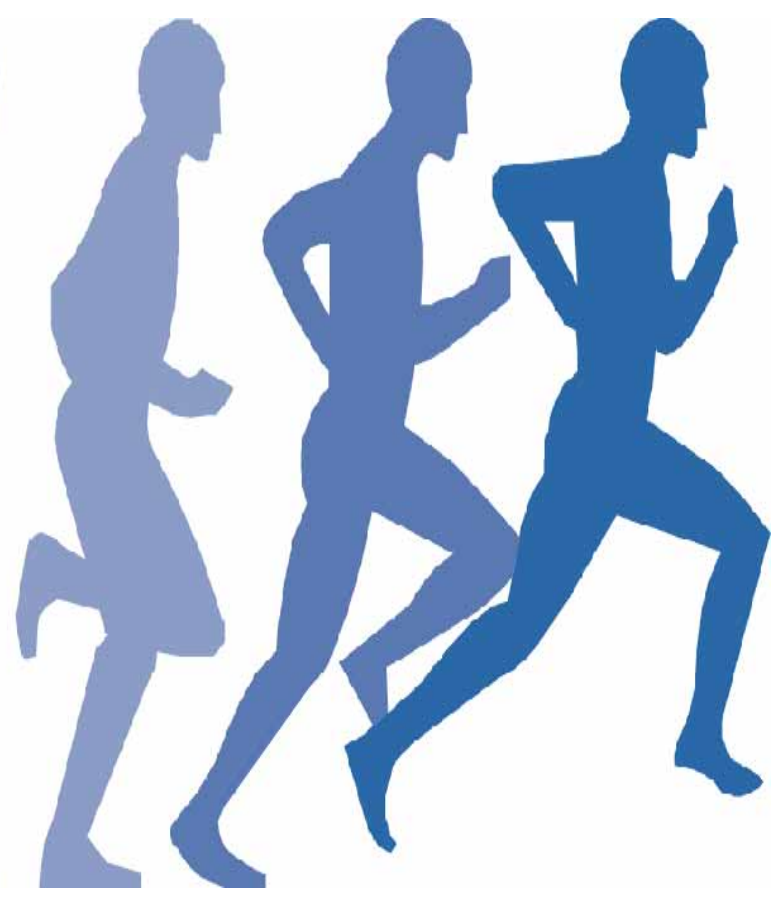

